\title{
Real-Time Optimization of Pharmacodynamic Target Attainment at Infection Site during Treatment of Post-Neurosurgical Ventriculitis Caused by Carbapenem-Resistant Gram Negatives with Ceftazidime-Avibactam-Based Regimens: A Report of Two Cases
}

\author{
Milo Gatti ${ }^{1,2}{ }^{\mathbb{D}}$, Giulio Virgili ${ }^{3}$, Pier Giorgio Cojutti ${ }^{2}$, Paolo Gaibani ${ }^{4} \mathbb{D}$, Matteo Conti $^{2} \mathbb{D}$, Carmelo Sturiale ${ }^{5}$, \\ Federico Pea ${ }^{1,2, *(\mathbb{D})}$ and Pierluigi Viale ${ }^{1,3}$
}

check for updates

Citation: Gatti, M.; Virgili, G.; Cojutti P.G.; Gaibani, P.; Conti, M.; Sturiale,

C.; Pea, F.; Viale, P. Real-Time Optimization of Pharmacodynamic Target Attainment at Infection Site during Treatment of Post-Neurosurgical Ventriculitis Caused by CarbapenemResistant Gram Negatives with Ceftazidime-Avibactam-Based Regimens: A Report of Two Cases. Microorganisms 2022, 10, 154. https://doi.org/10.3390/ microorganisms10010154 Academic Editor: Andrea Cortegian

Received: 9 December 2021 Accepted: 10 January 2022 Published: 12 January 2022 Publisher's Note: MDPI stays neutral with regard to jurisdictional claims in published maps and institutional affiliations.

Copyright: (C) 2022 by the authors. Licensee MDPI, Basel, Switzerland. This article is an open access article distributed under the terms and conditions of the Creative Commons Attribution (CC BY) license (https:// creativecommons.org/licenses/by/ $4.0 /)$.
1 Department of Medical and Surgical Sciences, Alma Mater Studiorum University of Bologna, 40138 Bologna, Italy; milo.gatti2@unibo.it (M.G.); pierluigi.viale@unibo.it (P.V.)

2 SSD Clinical Pharmacology, Department for Integrated Infectious Risk Management, IRCCS Azienda Ospedaliero-Universitaria di Bologna, 40138 Bologna, Italy; piergiorgio.cojutti@aosp.bo.it (P.G.C.); matteo.conti@ausl.imola.bo.it (M.C.)

3 Infectious Diseases Unit, Department for Integrated Infectious Risk Management, IRCCS Azienda Ospedaliero-Universitaria di Bologna, 40138 Bologna, Italy; giulio.virgili@aosp.bo.it

4 Division of Microbiology, IRCCS Azienda Ospedaliero-Universitaria di Bologna, 40138 Bologna, Italy; paolo.gaibani@unibo.it

5 Neurosurgery Unit, IRCCS Istituto delle Scienze Neurologiche Ospedale Bellaria di Bologna, 40319 Bologna, Italy; carmelo.sturiale@isnb.it

* Correspondence: federico.pea@unibo.it; Tel.: +39-051-214-3627

\begin{abstract}
We present two cases of post-neurosurgical ventriculitis caused by carbapenem-resistant Gram-negative pathogens successfully treated with high-dose ceftazidime/avibactam. The existence of a real-time clinical pharmacological advice program, by enabling the optimization of the PK/PD targets over time at the infection site, turned out to be very helpful.
\end{abstract}

Keywords: ceftazidime/avibactam; CSF penetration; post-neurosurgical ventriculitis; KPC-producing Klebsiella pneumoniae; carbapenem-resistant Pseudomonas aeruginosa; fosfomycin; real-time TDM-based clinical pharmacological advice

\section{Introduction}

Post-neurosurgical (post-NS) infections in neurocritical patients are associated with remarkable morbidity and mortality rates [1]. In recent years, several of these infections have been found to be increasingly caused by Gram-negative pathogens with a high level of resistance against most of the available antibiotics, and this makes the implementation of appropriate treatment extremely challenging [1].

Ceftazidime/avibactam is a novel beta-lactam/beta-lactamase inhibitor combination with valuable activity against carbapenemase-resistant (CR) Enterobacterales and/or Pseudomonas aeruginosa [2]. Nowadays, data concerning ceftazidime/avibactam use in difficult-to-treat CNS infections are limited to a few case series/reports [3]. Additionally, drug exposure into cerebrospinal fluid (CSF) was only assessed at a single time-point in a patient who received systemic administration of ceftazidime/avibactam via intermittent infusion [3], and continuous infusion (CI) was shown to provide significant benefits in terms of survival rate in patients with KPC-producing Klebsiella pneumoniae infections treated with ceftazidime/avibactam [4].

Here, we report two cases of post-NS infections caused by CR Gram negatives that were treated with high-dose CI ceftazidime/avibactam-based regimens. Treatment was 
optimized by means of a real-time clinical pharmacological advice (CPA) program focused at maximizing pharmacodynamic target attainment (PD-TA) at the infection site against CR pathogens that were isolated from the CSF.

\section{Case Presentation}

The first case was that of a 52-year-old male (who was being treated with norepinephrine, propofol, lansoprazole, enoxaparin, dexamethasone, baclofen, metoclopramide, venlafaxine, polyethylene glycol, and calcium gluconate) affected by post-traumatic cerebral ischemic injury that caused tetraplegia and hydrocephalus needing ventriculo-peritoneal shunt (VPS) positioning. During hospitalization, colonization with a CR Klebsiella pneumoniae (CR-KP) strain was documented at rectal swab, with susceptibility limited to ceftazidime/avibactam (MIC $4 \mathrm{mg} / \mathrm{L}$ ) and colistin (MIC $0.25 \mathrm{mg} / \mathrm{L}$ ). Five days later, an episode of CR-KP bacteraemia occurred, and treatment with ceftazidime/avibactam ( $2.5 \mathrm{~g}$ over $2 \mathrm{~h}$ loading dose (LD) followed by $2.5 \mathrm{~g}$ q8h over $8 \mathrm{~h}$ (namely by CI)) was started. CSF chemico-physical examination was carried out, and the findings were suggestive of VPS-related infection (protein $1.96 \mathrm{mg} / \mathrm{dL}$; glucose $1.0 \mathrm{mg} / \mathrm{dL}$; WBC count 17,695/ $\mathrm{mm}^{3}$, $84.4 \%$ of which being polymorphonuclear cells). Consequently, VPS was replaced by an external ventricular shunt (EVD), and linezolid co-treatment was added. Soon before VPS removal and soon after EVD positioning, CSF cultures were performed. Both samples yielded CR-KP strains with the same pattern of susceptibility, and consequently, linezolid was suspended. Additionally, a real-time CPA program based on plasma and CSF therapeutic drug monitoring (TDM) was implemented for maximizing the PD-TA of ceftazidime/avibactam in both plasma and CSF over time (desired targets: steady-state concentrations (Css)/MIC ratio 4-8 for ceftazidime and 100\%fT > $4 \mathrm{mg} / \mathrm{L}$ for avibactam). On day 4, both CSF pharmacodynamic targets were quasi-optimal (ceftazidime $\mathrm{C}_{\mathrm{ss}} / \mathrm{MIC}$ ratio (3.25) and avibactam CSF concentration (3.2 mg/L)) (Figure 1).

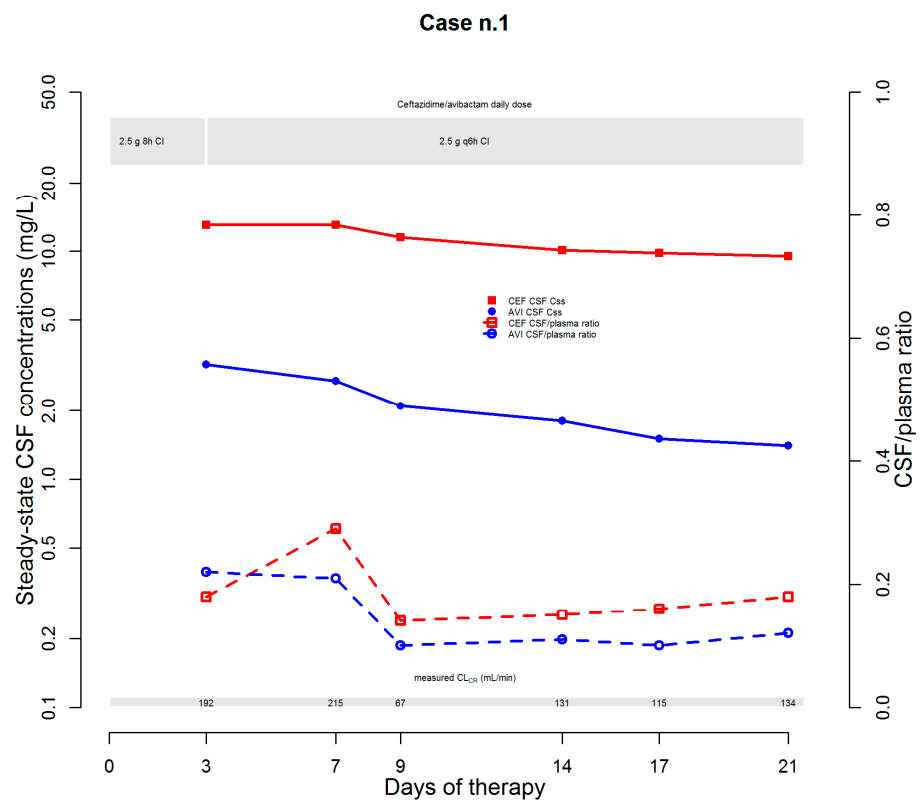

Figure 1. Temporal trends of steady-state CSF concentrations and of CSF/plasma ratio of ceftazidime and avibactam. AVI: avibactam; CEF: ceftazidime; $\mathrm{CI}$ : continuous infusion; $\mathrm{CL}_{\mathrm{CR}}$ : creatinine clearance; CSF: cerebrospinal fluid.

Ceftazidime/avibactam dosage was increased up to $2.5 \mathrm{~g}$ q6h over $6 \mathrm{~h} \mathrm{CI}$. The choice was supported by the finding of an augmented renal clearance at that time (measured CLCr of $192 \mathrm{~mL} / \mathrm{min}$ ) and by the contention that the progressive healing of the blood-brain barrier could have led to a decreased penetration rate of ceftazidime/avibactam into CSF [5]. Subsequent TDM assessments showed CSF-to-plasma ratios ranging between 0.14 and 
0.29 and between 0.10 and 0.22 for ceftazidime and avibactam, respectively. This approach allowed a quasi-optimal CSF PD-TA to be maintained over time (ceftazidime $\mathrm{C}_{\mathrm{ss}} / \mathrm{MIC}$ ratio ranging 2.38-3.25; avibactam concentrations ranging 1.4-2.7 mg/L). Subsequent CSF culture assessments showed microbiological eradication and culture negativization persisted over time, with favorable clinical evolution and progressive reduction in both protein levels (from 1964 to $42 \mathrm{mg} / \mathrm{dL}$ ) and WBC counts (from 17,695 to 3/ $\mathrm{mm}^{3}$ ). Although ceftazidime/avibactam treatment was successfully completed on day 21, unfortunately, the patient passed away because of underlying complications of the cerebral damage.

The second case was that of a 52-year-old male (who was being treated with lansoprazole, sertraline, levetiracetam, delorazepam, and enoxaparin) affected by a pineal neoplasm complicated by obstructive hydrocephalus. After neurosurgical exeresis, an EVS was positioned, and the post-NS course was complicated by fever coupled with headache relapse. CSF chemico-physical examination was suggestive of an EVS infection (protein $122.0 \mathrm{mg} / \mathrm{dL}$; WBCs $176 / \mathrm{mm}^{3}, 65.9 \%$ of which being polymorphonuclear cells). Empirical treatment with cefepime plus trimethoprim-sulfamethoxazole was started. On day 3, CSF culture was positive for a CR-Pseudomonas aeruginosa strain. The strain was susceptible to ceftazidime/avibactam (MIC $4 \mathrm{mg} / \mathrm{L}$ ), amikacin (MIC $8 \mathrm{mg} / \mathrm{L}$ ), and ceftolozane/tazobactam (MIC $1 \mathrm{mg} / \mathrm{L}$ ). Testing by agar dilution showed an MIC for fosfomycin of $64 \mathrm{mg} / \mathrm{L}$. After EVS removal and substitution, antibiotic therapy was consequently switched to ceftazidime/avibactam (2.5 g q6h over $6 \mathrm{~h} \mathrm{CI}$ after $2.5 \mathrm{LD})$ plus fosfomycin (8 g LD followed by $16 \mathrm{~g}$ q24h CI). A real-time CPA program was implemented for maximizing the PD-TAs of both ceftazidime/avibactam and fosfomycin into CSF (desired targets: for ceftazidime/avibactam, as previously mentioned; for fosfomycin, $f$ AUC / MIC ratio $\geq 40.8$ [6]). On day 2, CSF PD-TAs were mixed for ceftazidime/avibactam (ceftazidime $\mathrm{C}_{\mathrm{ss}} / \mathrm{MIC}$ ratio 1.7 , avibactam concentration $4.1 \mathrm{mg} / \mathrm{L}$ ) and borderline for fosfomycin ( $f$ AUC / MIC ratio 50.3 (Figure 2)).

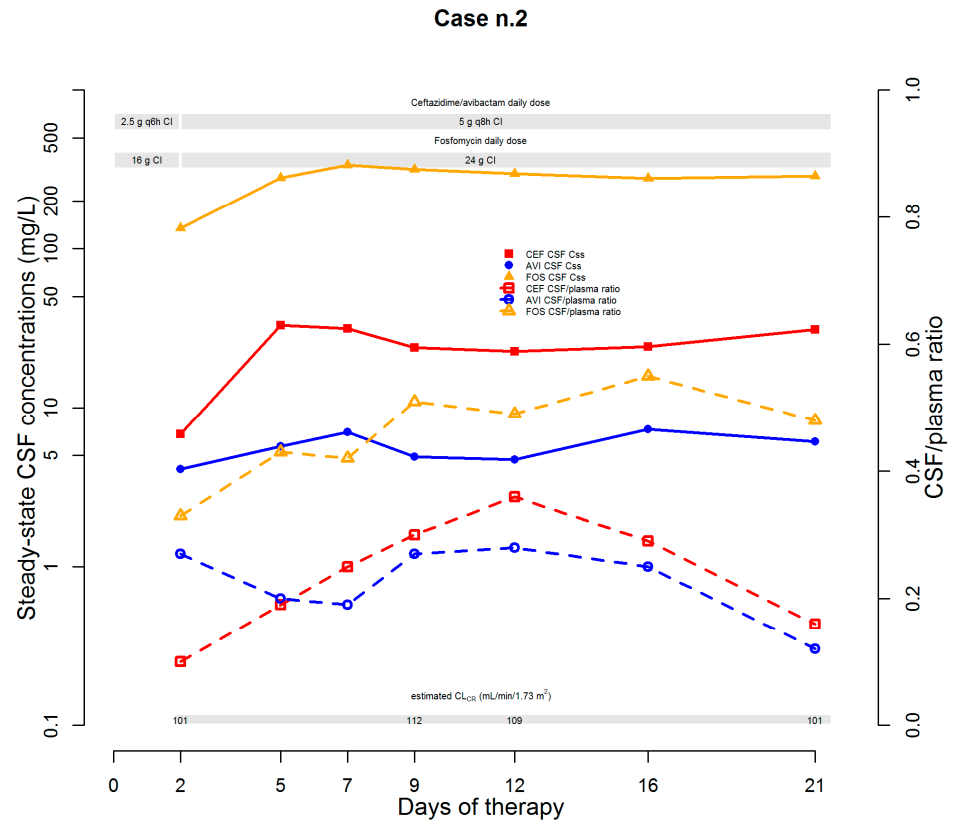

Figure 2. Temporal trends of steady-state CSF concentrations and of CSF/plasma ratio of ceftazidime/avibactam and fosfomycin. AVI: avibactam; CEF: ceftazidime; CI: continuous infusion; $\mathrm{CL}_{\mathrm{CR}}$ : creatinine clearance; CSF: cerebrospinal fluid; FOS: fosfomycin.

Estimated CLCr was $101 \mathrm{~mL} / \mathrm{min} / 1.73 \mathrm{~m}^{2}$, and dosages of both antibiotics were increased (up to $5 \mathrm{~g}$ q8h over $8 \mathrm{~h} \mathrm{CI}$ for ceftazidime/avibactam and $24 \mathrm{~g}$ q24h CI for fosfomycin). This choice was also supported by the contention that the progressive healing of the blood-brain barrier could have led to a decrease in the penetration rate of both antibiotics into CSF [5]. Subsequent CSF assessments showed a CSF-to-plasma ratio 
ranging between 0.16 and 0.36 and between 0.12 and 0.28 for ceftazidime and avibactam, respectively. PD-TAs of both ceftazidime and avibactam were always optimal (ceftazidime $\mathrm{C}_{\mathrm{ss}} / \mathrm{MIC}$ ratio ranging from 5.675 to 8.25 and avibactam concentrations ranging from 4.7 to $7.3 \mathrm{mg} / \mathrm{L}$ ). For fosfomycin, CSF-to-plasma ratios ranged between 0.42 and 0.5 , and the CSF PD-TAs were always optimal as well ( $f$ AUC/MIC ratio range 104.63-126.38). Subsequent CSF assessments showed microbiological eradication and culture negativization persisted over time, with favorable clinical evolution and progressive reductions in both protein levels and WBC counts. EVS was removed on day 23 and antibiotic therapy was stopped on day 26 after achieving optimal clinical response. The patient was discharged to a rehabilitation facility, and no relapse at follow-up occurred.

\section{Discussion}

Overall, these two cases firstly showed that the CSF penetration rates of ceftazidime and avibactam in patients with post-NS infections ranged over time around $15-30 \%$ during the first 3 weeks post-event. Additionally, our findings showed that optimizing CSF PDTAs by means of a real-time CPA program may be valuable in tackling CR-related post-NS infections caused by borderline susceptible pathogens. Repeating TDM assessments over time may also be crucial in neurosurgical patients. This approach may allow the posology to be modulated day-by-day according to the dynamic evolution of the CNS infection [5] and/or to the fluctuations of renal function, given that $\mathrm{ARC}$ is a highly probable occurrence in the early post-event phase of neurocritical patients [7]. Furthermore, it may also concur in avoiding the need for intrathecal administration of antibiotics that could be complicated by potential CNS adverse events. Notably, in the patient who experienced ARC, treatment with ceftazidime/avibactam doses up to 10-15 g/day did not cause any adverse event. Finally, it should be mentioned that the choice of combining ceftazidime/avibactam with fosfomycin for treating CR-P. aeruginosa related post-NS infection was based on preclinical studies showing synergism. In a murine infection model using a high bacterial burden, the combination of ceftazidime/avibactam with fosfomycin was superior to either drug alone in significantly reducing the P. aeruginosa burden [8]. Notably, the high CSF penetration rate of fosfomycin (45-50\%) allowed optimal PD-TAs over time in our patient by administering doses up to $24 \mathrm{~g}$ q $24 \mathrm{~h} \mathrm{CI}$.

In conclusion, our findings showed that the existence of a real-time CPA program, by enabling optimization of the PK/PD targets over time at the infection site, turned out to be very helpful in granting successful treatment with high-dose CI ceftazidime/avibactambased regimens of two cases of post-NS ventriculitis caused by CR-Gram-negative pathogens. Of course, ours is simply a proof-of-concept and we are aware that further prospective confirmatory studies are warranted before any definitive conclusion can be drawn.

Author Contributions: Conceptualization, M.G. and F.P.; methodology, M.G., P.G.C. and F.P.; data curation, M.G., G.V., P.G., M.C. and C.S.; writing-original draft preparation, M.G. and G.V.; writingreview and editing, F.P. and P.V. All authors have read and agreed to the published version of the manuscript.

Funding: This research received no external funding.

Institutional Review Board Statement: Not applicable.

Informed Consent Statement: Not applicable.

Data Availability Statement: The data presented in this study are available on request from the corresponding author. The data are not publicly available due to privacy concerns.

Conflicts of Interest: M.G. reports grants from Angelini S.p.A., outside the submitted work. F.P. reports personal fees from Angelini, Basilea Pharmaceutica, Gilead, Hikma, MSD, Pfizer, SanofiAventis, Shionogi, Thermo Fisher, and Accelerate Diagnostics, outside the submitted work; has participated in speaker's bureau for Accelerate Diagnostics, Angelini, Basilea Pharmaceutica, Gilead, Hikma, MSD, Pfizer, Sanofi-Aventis, Shionogi, Thermo Fisher, and as consultant for Angelini, Basilea Pharmaceutica, Gilead, MSD, Pfizer, Shionogi, outside the submitted work. P.V. has served as a 
consultant for bioMérieux, Gilead, Merck Sharp and Dohme, Nabriva, Nordic Pharma, Pfizer, ThermoFisher, and Venatorx, and received payment for serving on the speaker's bureaus for Correvio, Gilead, Merck Sharp and Dohme, Nordic Pharma, and Pfizer, outside the submitted work. The other authors report no potential conflict of interest for this work.

\section{References}

1. Karvouniaris, M.; Brotis, A.G.; Tsiamalou, P.; Fountas, K.N. The Role of Intraventricular Antibiotics in the Treatment of Nosocomial Ventriculitis/Meningitis from Gram-Negative Pathogens: A Systematic Review and Meta-Analysis. World Neurosurg. 2018, 120, e637-e650. [CrossRef] [PubMed]

2. Soriano, A.; Carmeli, Y.; Omrani, A.S.; Moore, L.S.P.; Tawadrous, M.; Irani, P. Ceftazidime-Avibactam for the Treatment of Serious Gram-Negative Infections with Limited Treatment Options: A Systematic Literature Review. Infect. Dis. Ther. 2021, 10, 1989-2034. [CrossRef] [PubMed]

3. Yasmin, M.; Hanrahan, J.; Marshall, S.; Lodise, T.P.; Chen, L.; Perez, F.; Kreiswirth, B.; Bonomo, R.A. Using Therapeutic Drug Monitoring to Treat KPC-Producing Klebsiella Pneumoniae Central Nervous System Infection with Ceftazidime/Avibactam. Open Forum Infect. Dis. 2020, 7, ofaa349. [CrossRef] [PubMed]

4. Tumbarello, M.; Raffaelli, F.; Giannella, M.; Mantengoli, E.; Mularoni, A.; Venditti, M.; De Rosa, F.G.; Sarmati, L.; Bassetti, M.; Brindicci, G.; et al. Ceftazidime-Avibactam Use for KPC-Kp Infections: A Retrospective Observational Multicenter Study. Clin. Infect. Dis. 2021, 73, 1664-1676. [CrossRef] [PubMed]

5. Lutsar, I.; McCracken, G.H.; Friedland, I.R. Antibiotic Pharmacodynamics in Cerebrospinal Fluid. Clin. Infect. Dis. 1998, 27, 1117-1127. [CrossRef] [PubMed]

6. Lepak, A.J.; Zhao, M.; VanScoy, B.; Taylor, D.S.; Ellis-Grosse, E.; Ambrose, P.G.; Andes, D.R. In Vivo Pharmacokinetics and Pharmacodynamics of ZTI-01 (Fosfomycin for Injection) in the Neutropenic Murine Thigh Infection Model against Escherichia Coli, Klebsiella Pneumoniae, and Pseudomonas Aeruginosa. Antimicrob. Agents Chemother. 2017, 61, e00476-17. [CrossRef] [PubMed]

7. Cook, A.M.; Hatton-Kolpek, J. Augmented Renal Clearance. Pharmacotherapy 2019, 39, 346-354. [CrossRef] [PubMed]

8. Papp-Wallace, K.M.; Zeiser, E.T.; Becka, S.A.; Park, S.; Wilson, B.M.; Winkler, M.L.; D’Souza, R.; Singh, I.; Sutton, G.; Fouts, D.E.; et al. Ceftazidime-Avibactam in Combination with Fosfomycin: A Novel Therapeutic Strategy Against Multidrug-Resistant Pseudomonas Aeruginosa. J. Infect. Dis. 2019, 220, 666-676. [CrossRef] [PubMed] 